\title{
Limb lengthening with one Precice nail over its capacity
}

\author{
Mesut Kariksiz, MD, Ozgur Karakoyun, $M D$.
}

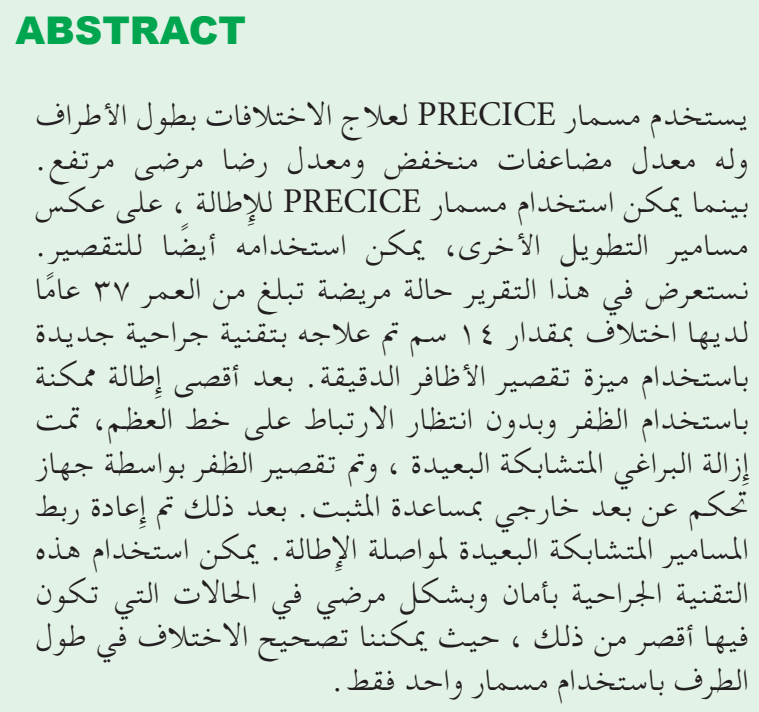

The Precice nail is used to treat limb length discrepancies and has a low complication rate and a high patient satisfaction rate. While the Precice nail can be used for lengthening, unlike other lengthening nails, it can also be used for shortening. We report a 37 -year-old female patient with a $14 \mathrm{~cm}$ limb length discrepancy that was treated with a new surgical technique using the shortening feature of the Precice nail. Following maximum possible lengthening using the nail and without waiting for union on the osteotomy line, the distal interlocking screws were removed, and the nail was shortened by external remote control with the help of the fixator. The distal interlocking screws were then reattached to continue lengthening. This surgical technique can be used safely and satisfactorily in cases with more shortness as we can correct the extremity length discrepancy using only one nail.

Saudi Med J 2019; Vol. 40 (10): 1058-1062 doi: 10.15537/smj.2019.10.24019

From the Department of Orthopedics and Traumatology (Kariksiz), Gazi Yasargil Training and Research Hospital, Diyarbakir and from the Orthopedics Clinics (Karakoyun), Tekirdag, Turkey.
Received 26th February 2019. Accepted 24th July 2019.

Address correspondence and reprint request to: Dr. Mesut Kariksiz, Department of Orthopedics and Traumatology, Gazi Yasargil Training and Research Hospital, Diyarbakir, Turkey.E-mail: mesutkariksiz@gmail.com ORCID: https://orcid.org/0000-0002-9632-7694

$\mathrm{E}$ xtremity lengthening is safely performed using a distraction osteogenesis technique. This technique is currently mostly performed using external fixators. External fixator applications have some disadvantages; however, including pin site infection, joint stiffness, contractures, cosmetic problems, and fractures following fixator removal. ${ }^{1}$ To reduce these complications, novel methods have been developed in which a distraction step is performed using an external fixator, followed by a consolidation step using a nail or plate. Accordingly, surgeons have attempted to shorten the period for which the external fixator stays on the patient's body. ${ }^{2}$ With advancing technologies, intramedullary lengthening nails have started to be produced. Among these, the Albizzia intramedullary nail and the intramedullary skeletal kinetic distractor (ISKD) provide mechanical lengthening, while the Fitbone nail provides lengthening via a motorized mechanism and the Precice nail via magnetic lengthening. ${ }^{3,4}$ The Precice nail consists of a magnetic metal shaft and gears attached together. This mechanism is activated by an external remote control (ERC) on the skin. Unlike other lengthening nails, both lengthening and shortening can be performed using the ERC. The lengthening capacity of the currently available Precice nails is $50 \mathrm{~mm}$ for the nails measuring less than $245 \mathrm{~mm}$ and $80 \mathrm{~mm}$ for the nails that are $245 \mathrm{~mm}$ and over. Each $1 \mathrm{~mm}$ lengthening or shortening takes approximately 7 minutes.

We believe this surgical technique is the first described limb lengthening using a single Precice nail

Disclosure. Authors have no conflict of interests, and the work was not supported or funded by any drug company. 
in which the shortening feature was used to reset the nail for further lengthening.

Case Report. Patient's information. A 37-year-old female patient was admitted to our clinic with a $14 \mathrm{~cm}$ discrepancy of the right lower extremity. The etiology of the shortening was congenital pseudarthrosis of the tibia for which the union had been obtained with a circular external fixator at another center. In the past, successful arthrodesis of the left ankle of the patient had also been performed (Figures 1A \& 1B).

Clinical findings. The left tibia measurement was $18 \mathrm{~cm}$, and the medulla was $8 \mathrm{~mm}$ at its narrowest site on the direct $\mathrm{x}$-rays (Figures $1 \mathrm{C} \& 1 \mathrm{D}$ ). The knee had a full range of motion (ROM).
Diagnostic assessment. Direct $\mathrm{x}$-ray and orthorontgenogram were used.

Therapeutic intervention. The tibia was reamed up to $9 \mathrm{~mm}$ with a flexible reamer, and a $185 \mathrm{~mm} \mathrm{x}$ $8.5 \mathrm{~mm}$ Precice nail was inserted into the right tibia under anesthesia (Figures 2A \& 2B). A proximal tibial osteotomy and a fibular osteotomy were performed using a mini incision and multiple drill technique. After implanting the nail, the first distraction of $1 \mathrm{~mm}$ was performed with the patient under anesthesia. On the 6th postoperative day, the patient was taught to lengthen the nail by $0.33 \mathrm{~mm} 3$ times per day using the ERC. The lengthening of the nail was completed on the 56th postoperative day (Figures 2C \& 2D). Thereafter, a unilateral external fixator was applied using one Schanz screw each for the proximal and distal parts of the
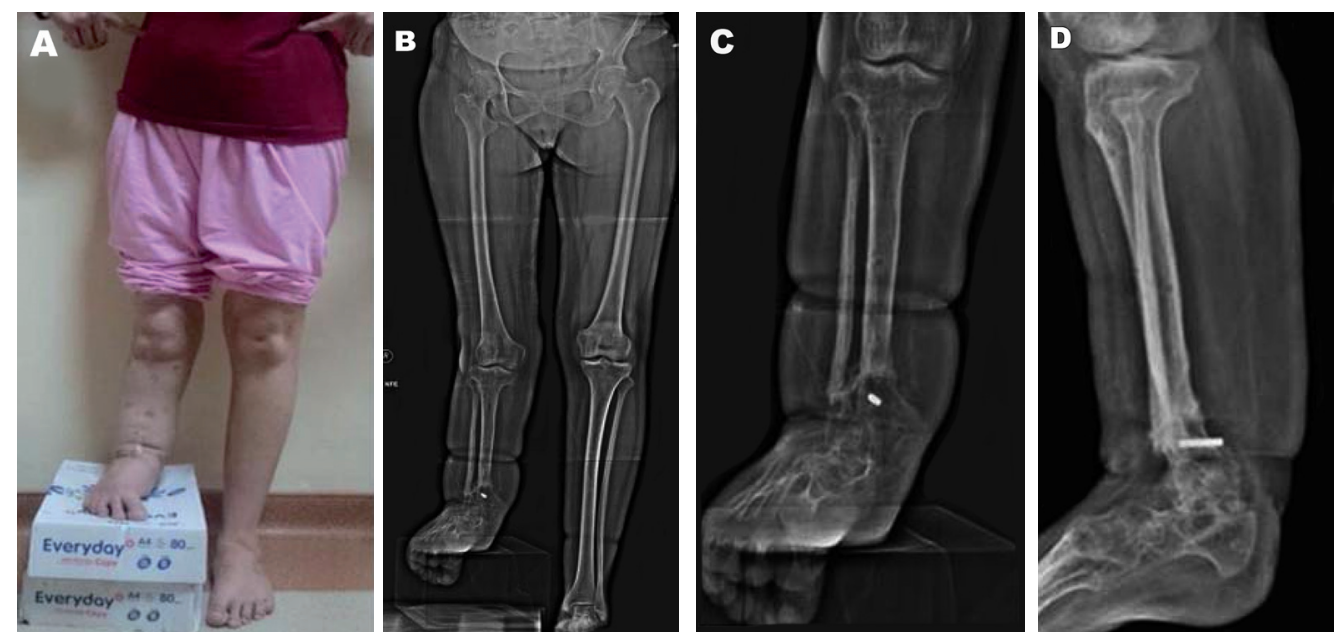

Figure 1 - The preoperative view of the patient (A), preoperative orthoroentgenogram (B), preoperative anteroposterior direct X-ray view (C), and preoperative lateral x-ray view (D).
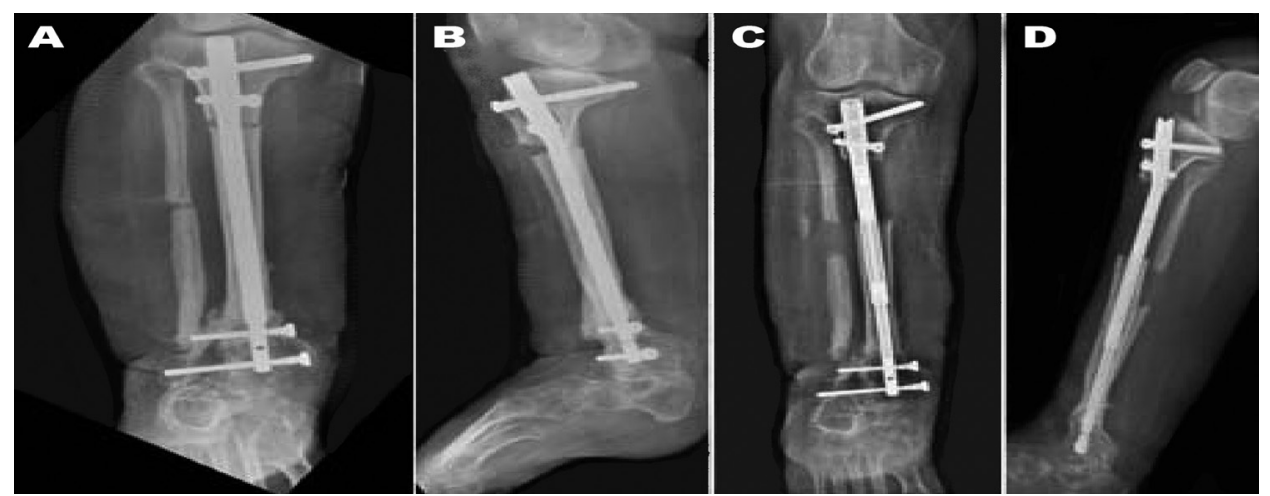

Figure 2 - The Precise nail was inserted into the right tibia under anesthesia: anteroposterior direct x-ray view (A), lateral x-ray view (B). After $50 \mathrm{~mm}$ of lengthening had been completed: anteroposterior direct $\mathrm{x}$-ray view $(\mathrm{C})$, and lateral $\mathrm{x}$-ray view (D). 
tibia, and the distal interlocking screw was removed under anesthesia (Figures 3A \& 3B). We opted not to retrieve the nail under anesthesia during the operation because it would have taken 350 minutes to completely remove the nail. The first part of the procedure was completed, and the patient was taken back to her room. The nail was shortened by $25 \mathrm{~mm}$ per day over 2 days by the patient with the ERC in manual mode (Figures 3C \& 3D). After the nail shortening had been completed, the distal interlocking screws were placed, and the unilateral external fixator was removed under anesthesia (Figures 4A \& 4B). Lengthening was started again on the first postoperative day, and the second lengthening period was completed in 50 days. A total lengthening of $100 \mathrm{~mm}$ was obtained with the nail, the overall capacity of which allowed for a maximum of 50 mm lengthening (Figures $4 \mathrm{C} \& 4 \mathrm{D}$ ).

Follow-up and outcomes. The follow-up period with the patient was 49 months. Successful limb lengthening and bone healing were achieved. Table 1 represents the timeline from patient's treatment process.

Discussion. External fixators using the distraction osteogenesis method are still the most frequently used orthopedic instruments for correcting length discrepancies in lower limbs. On the other hand, lengthening operations using external fixators come with many different types of complications. ${ }^{5}$ Pin site infections, deep soft tissue infections, and pain due to Schanz nails and Kirschner wires are the most commonly seen problems in clinical practice and reduce postoperative patient satisfaction. These complications can be precluded by performing extremity lengthening
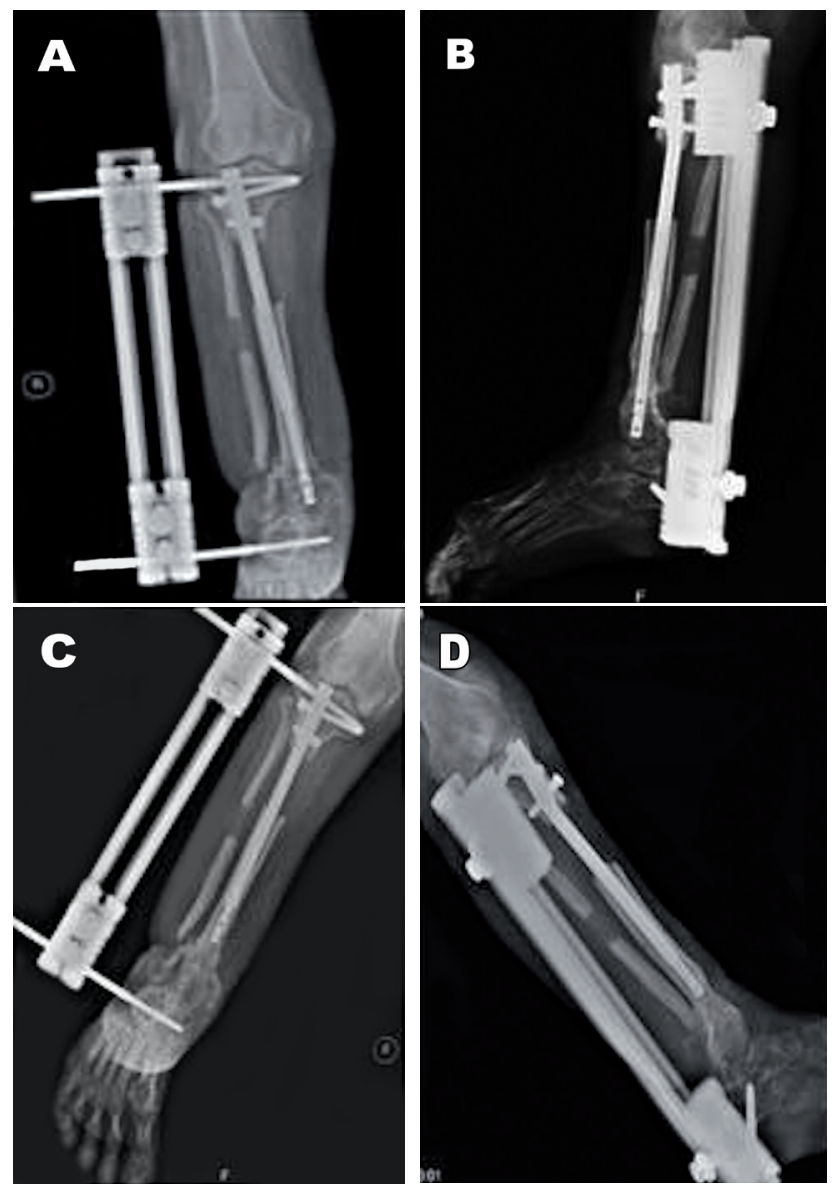

Figure 3 - A unilateral external fixator was applied using one Schanz screw each for the proximal and distal parts of the tibia, and the distal interlocking screw was removed under anesthesia: anteroposterior direct $\mathrm{x}$-ray view (A) and lateral $\mathrm{x}$-ray view (B). The patient used the external remote control in manual mode to shorten the nail by $25 \mathrm{~mm}$ per day over 2 days: anteroposterior direct $\mathrm{x}$-ray view $(\mathrm{C})$, lateral $\mathrm{x}$-ray view $(\mathrm{D})$.

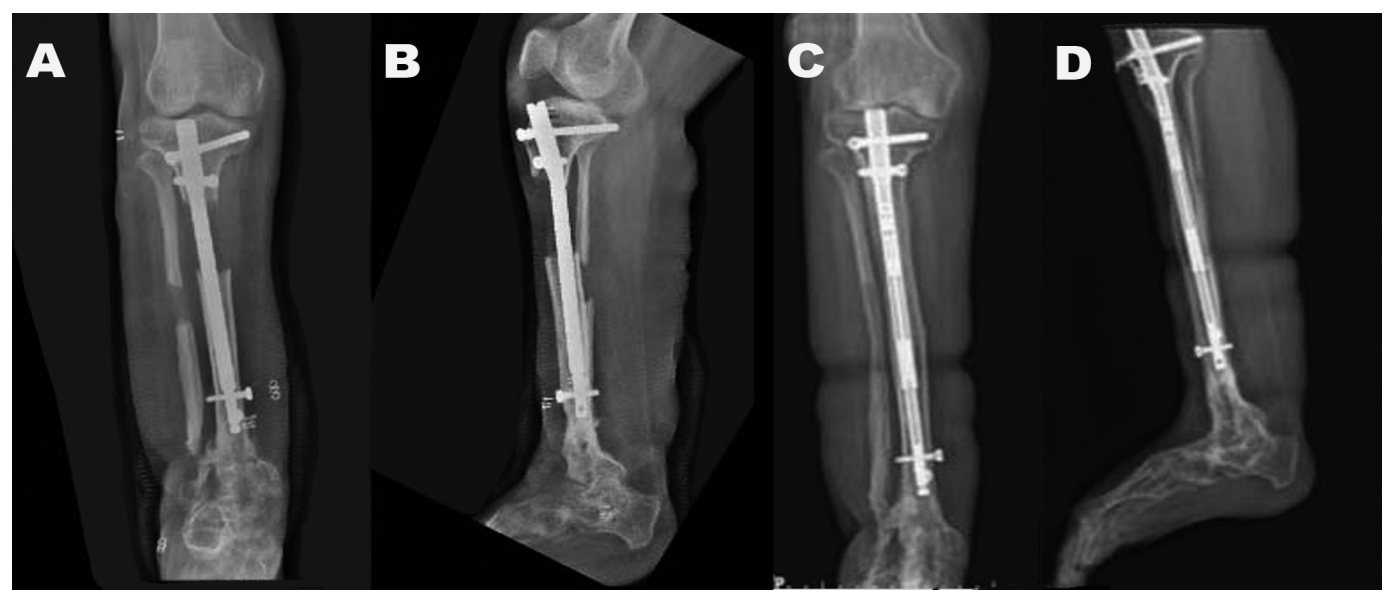

Figure 4 - Once the nail shortening was complete, the distal interlocking screws were placed, and the unilateral external fixator was removed under anesthesia: anteroposterior direct $\mathrm{x}$-ray view (A) and lateral $\mathrm{x}$-ray view (B). A total lengthening of $100 \mathrm{~mm}$ was obtained with the nail, the maximum capacity of which allows for $50 \mathrm{~mm}$ of lengthening: anteroposterior direct X-ray view (C) and lateral X-ray view (D). 
Table 1 - Timeline of a 37-year-old female patient was admitted to our clinic with a $14 \mathrm{~cm}$ discrepancy of the right lower extremity. The etiology of the shortening was congenital pseudarthrosis of the tibia for which the union had been obtained with a circular external fixator at another center. In the past, successful arthrodesis of the left ankle of the patient had also been performed.

\begin{tabular}{ll}
\hline Date & \multicolumn{1}{c}{ Intervention } \\
\hline 11 Nov 2014 & $\begin{array}{l}\text { Initial evaluation and preparation of surgery } \\
\text { 19 Nov 2014 } \\
\text { lengery (insertion of lengthening nail and start } \\
\text { 19 Jan 2015 }\end{array}$ \\
Surgery (fixator insertion, removal of distal locking \\
screws and shortening of the nail) \\
Surgery (fixator removal, inserting distal locking screws \\
12 Mar 2015 & $\begin{array}{l}\text { Completion of 10 cm lengthening } \\
\text { 15 Dec 2018 }\end{array}$ \\
\hline
\end{tabular}

using the Precice nail. In our case, the patient did not experience superficial or deep infection.

Joint contracture problems are commonly seen with extremity lengthening using external fixators. In extremity lengthening operations using the Precice nail, the patient can start doing joint ROM exercises on the 1 st postoperative day. Our patient, who started ROM exercises during the early postoperative period, did not develop joint contracture. Her fused ankle prevented equinus contracture, which needs to be considered with an intact ankle. We only allowed partial weight bearing until we observed union in 3 cortices.

Nail arrest and mechanical problems with the Precice nail have been reported in some studies. ${ }^{6}$ Lengthening and shortening of the nail to the end of its capacity may cause problems in the nail mechanism during the second lengthening. In our case, no mechanical problems or nail arrest were developed.

Tibial-lengthening patients have reportedly needed to undergo a further osteotomy in the fibula due to early fibular union. ${ }^{7}$ As a $1 \mathrm{~cm}$ resection was performed in the fibula after the osteotomy in our case, no early union was noted. İt is generally accepted that the fibula should be transfixed for large lengthening of the tibia, but we did not transfix the fibula in this case due to ankle arthrodesis.

Our patient had a distraction index of $1.08 \mathrm{day} / \mathrm{mm}$ and a bone-healing index of $27 \mathrm{day} / \mathrm{cm}$, which is consistent with the literature. ${ }^{8}$ The patient's postoperative medial axis deviation (MAD) was determined to be within functional limits. We also found the bone and functional scores used by Paley for patients undergoing intramedullary nail lengthening to be excellent.
The osteotomy site was very proximal. If the osteotomy had been made from below, we would not have had enough space for the second lengthening. This would have caused the proximal tibia to flex at the time of nail insertion; however, it did not seem to flex during lengthening in our case. The tibia could otherwise be expected to bend into the valgus during lengthening. The lateral nail start point may have prevented this complication.

The shortening feature of the Precice nail is also used for compression in the case of nonunion and shortening where there is over-distraction. Using the Precice nail, André Couto et $\mathrm{al}^{9}$ removed the distal interlocking screws and shortened the nail after the completion of union of the osteotomy region in a patient with a short femur and continued lengthening after performing another osteotomy in the following step and locking the nail. Wozasek \& $\mathrm{Zak}^{10}$ conducted fixator-assisted lengthening with the Precice nail in a patient with humerus shortness. We did not wait for union in our patient. After completing $50 \mathrm{~mm}$ of lengthening, we shortened the nail by applying an external fixator and then started nail lengthening again. A second osteotomy was therefore not necessary. Furthermore, since we did not wait for the union of the osteotomy region, the treatment period was shorter.

In cases where it is not possible to correct extremity discrepancies using a single nail, we mostly use a longer, second nail after removing the first one. The treatment costs of the technique that we developed were considerably lower compared to the cost of treatment with multiple nails.

The surgical technique that we have described can be used safely and satisfactorily for patients with more shortness as surgeons can correct the extremity length discrepancy using only one nail. In the future, we hope to perform similar studies with more patients.

\section{References}

1. Paley D. Problems, obstacles, and complications of limb lengthening by Ilizarov technique. Clin Orthop Relat Res 1990; (250): 81-104.

2. Bernstein M, Fragomen AT, Sabharwal S, Barclay J, Rozbruch SR. Does integrated fixation provide benefit in the reconstruction of posttraumatic tibial bone defects? Clin Orthop Relat Res 2015; 473: 3143-3153.

3. Guichet JM, Deromedis B, Donnan LT. Gradual femoral lengthening with the albizziai intramedullery nail. J Bone Joint Surg Am 2003; 85: 838-848.

4. Kucukkaya M, Karakoyun Ö, Sökücü S, Soydan R. Femoral lengthening and deformity correction using the fitbone motorized lengthening nail. J Orthop Sci 2015; 20: 149-154. 
5. Tjernstöm B, OLerud S, Rehnberg L. Limb lengthening by callus distraction. Complications in 53 cases operated 1980-1991. Acta Orthop Scand 1994; 65: 447-455.

6. Kirane YM, Fragomen AT, Rozbruch SR. Precision of the PRECICE internal bone lengthening nail. Clin Orthop Relat Res 2014; 472: 3869-3878.

7. Thaller PH, Furmetz J, Wolf F, Eilers T, Mutschler W. Limb lengthening with fully implantable magnetically actuated mechanical nails (PHENIX $((\mathrm{R}))$-preliminary results. Injury 2014; 45 (Suppl 1): S60-S65.
8. Kirane YM, Fragomen AT, Rozbruch SR. Precision of the PRECICE ${ }^{\circledast}$ internal bone lengthening nail. Clin Orthop Relat Res 2014; 472: 3869.

9. André Couto, Joana Freitas, Nuno Alegrete, Jorge Coutinho, Gilberto Costa. Two consecutive limb lengthening with the same PRECICE nail: a technical note. Strategies Trauma Limb Reconstr 2018; 13: 199-204.

10. Gerald E. Wozasek, Lukas Zak. Intramedulläre Oberarmverlängerung. Der Unfallchirurg 2018; 121: 868-873.

\section{Case Reports}

Case reports will only be considered for unusual topics that add something new to the literature. All Case Reports should include at least one figure. Written informed consent for publication must accompany any photograph in which the subject can be identified. Figures should be submitted with a 300 dpi resolution when submitting electronically. The abstract should be unstructured, and the introductory section should always include the objective and reason why the author is presenting this particular case. References should be up to date, preferably not exceeding 15 . 